\title{
Feasibility Analysis of Real-Time Periodic Tasks with Offsets
}

RODOLFO PELLIZZONI

GIUSEPPE LIPARI

rodolfo@sssup.it

Scuola Superiore S. Anna, Pisa, Italy

\begin{abstract}
The problem of feasibility analysis of asynchronous periodic task sets, where tasks can have an initial offset, is known to be co-NP-complete in the strong sense. A sufficient pseudo-polynomial test has been proposed by Baruah, Howell and Rosier, which consists in analyzing the feasibility of the corresponding synchronous task set (i.e. all offsets are set equal to 0). If the test gives a positive result, then the original asynchronous task set is feasible; else, no definitive answer can be given. In many cases, this sufficient test is too pessimistic, i.e. it gives no response for many feasible task sets.

In this paper, we present a new sufficient pseudo-polynomial test for asynchronous periodic task sets. Our test reduces the pessimism by explicitely considering the offsets in deriving a small set of critical arrival patterns. We show, trough a set of extensive simulations, that our test outperforms the previous sufficient test.
\end{abstract}

Keywords: real-time systems, scheduling

\section{Introduction}

In critical real-time systems it is necessary to guarantee a priori that all timing constraints are respected. Usually, these systems are modelled as sets of concurrent periodic real-time tasks. Each task $\tau_{i}$ is characterized by an initial offset $\phi_{i}$, a worst-case execution time $C_{i}$, a relative deadline $D_{i}$ and a period $T_{i}$. In general, a task's relative deadline can be different from its period. At run time, tasks are executed on the system by a real-time operating system. The scheduling algorithm selects which task to execute at each time instant, generating a schedule.

A feasible schedule is a schedule in which all tasks meet their deadlines and any additional specified constraint. A feasibility test is an algorithm that, given a task set, returns a positive answer if there exists a feasible schedule. In case of negative answer, no algorithm can generate a feasible schedule. A schedulability test is an algorithm that, given a task set and a scheduling algorithm, returns a positive answer if the algorithm generates a feasible schedule.

In single preemptive processor systems, the Earliest Deadline First (EDF) scheduling algorithm is optimal (Dertouzos, 1974), in the sense that: if a task set is feasible, then it is schedulable by EDF. Therefore, the feasibility problem on single processor systems can be reduced to the problem of testing the schedulability with EDF.

The feasibility problem for a set of independent periodic tasks to be scheduled on a single processor has been proven to be co-NP-complete in the strong sense (Leung and Merril, 1980; Baruah et al., 1990b). In Leung and Merril (1980) the authors showed that it 
is necessary to analyze all deadlines from 0 to $\Phi+2 H$ where $\Phi$ is the largest task offset and $H$ is the hyperperiod (i.e. the least common multiple of all task periods). In Baruah et al. (1990b) it is proved that, when the system utilization $U=\sum_{i=1}^{N} \frac{C_{i}}{T_{i}}$ (where $N$ is the number of tasks) is strictly less than 1, the Leung and Merril's condition is also sufficient.

Under certain assumptions, the problem becomes more tractable. For example, if deadlines are equal to periods, a simple polynomial test has been proposed (Liu and Layland, 1973). If the system utilization $U$ is less than or equal to 1 , the system is schedulable:

$$
U=\sum_{i=1}^{N} \frac{C_{i}}{T_{i}} \leq 1
$$

If the deadlines are less than or equal to the periods and the task set is synchronous (i.e. all tasks have initial offset equal to 0), then a pseudo-polynomial test has been proposed (Baruah et al., 1990a, 1990b).

In the case of asynchronous periodic task sets, any known necessary and sufficient feasibility test requires an exponential time to run. However, it is possible to obtain a sufficient test by ignoring the offsets and considering the task set as synchronous. In Baruah et al. (1990b) the authors showed that, given an asynchronous periodic task set $\mathcal{T}$, if the corresponding synchronous task set $\mathcal{T}^{\prime}$ (obtained by considering all offsets equal to 0 ) is feasible, then $\mathcal{T}$ is feasible too. However, if $\mathcal{T}^{\prime}$ is not feasible, no definitive answer can be given on $\mathcal{T}$. In some cases this sufficient test is quite pessimistic, as we will show in Section 5.

The basic idea behind Baruah's result is based on the concept of busy period. A busy period is an interval of time where the processor is never idle. If all tasks start synchronously at the same time $t$, the first deadline miss (if any) must happen in the longest busy period starting from $t$. When tasks have offsets, it may not be possible for them to start at the same time. Hence, we do not know where the deadline miss might happen in the schedule.

In this paper, a new sufficient pseudo-polynomial feasibility test for asynchronous task sets is proposed. Our idea is based on the observation that the patterns of arrivals of the tasks depend both on the offsets and on the periods of the tasks. By computing the minimum possible distance between the arrival times of any two tasks, we are able to select a small group of critical arrival patterns that generate the worst-case busy period. Our arrival patterns are pessimistic, in the sense that some of these patterns may not be possible in the schedule. Therefore, our test is only sufficient. However, experiments show that our test greatly reduces pessimism with respect to previous sufficient tests.

\section{System Model}

In this paper, the problem of feasibility analysis of a periodic task set on a single processor is considered. We assume that all task parameters are expressed by integer numbers. Time is divided into slots, numbered starting from $0: t \in \mathbb{N}$.

The system consists of a set $\mathcal{T}$ of $N$ periodic real time tasks $\tau_{1}, \ldots, \tau_{N}$. A task $\tau_{i}$ is characterized by a tuple $\left(\phi_{i}, C_{i}, D_{i}, T_{i}\right)$ where $\phi_{i}$ is the offset, $C_{i}$ is the worst case computation time, $D_{i}$ is the relative deadline and $T_{i}$ is the period. Each task $\tau_{i}$ generates an 
infinite number of jobs $\tau_{i j}, j \geq 0$. Each job is assigned a release time $r_{i j}=\phi_{i}+j T_{i}$ and an absolute deadline $d_{i j}=r_{i j}+D_{i}$.

A task set $\mathcal{T}$ is said to be synchronous if all offsets are equal to zero. A task set is said to be asynchronous if it isn't synchronous. Given an asynchronous task set $\mathcal{T}=\left\{\tau_{1}, \ldots, \tau_{N}\right\}$, we define the corresponding synchronous task set as $\mathcal{T}^{\prime}=\left\{\tau_{1}^{\prime}, \ldots, \tau_{N}^{\prime}\right\}$, where for every task $\tau_{i}^{\prime}: \phi_{i}^{\prime}=0, C_{i}^{\prime}=C_{i}, D_{i}^{\prime}=D_{i}, T_{i}^{\prime}=T_{i}$.

We further define:

1. $U_{i}=\frac{C_{i}}{T_{i}}$ is the utilization of task $\tau_{i}$;

2. $U=\sum_{i=1}^{N} U_{i}$ is the total utilization of task set $\mathcal{T}$;

3. $\Phi=\max \left\{\phi_{1}, \ldots, \phi_{N}\right\}$ is the largest offset;

4. function $\operatorname{gcd}\left(T_{i}, T_{j}\right)$ is the greatest common divisor between two periods $T_{i}$ and $T_{j}$;

5. function $\operatorname{lcm}\left(T_{1}, \ldots, T_{j}\right)$ is the least common multiple among all periods $T_{1}, \ldots, T_{j}$;

6. $H=\operatorname{lcm}\left\{T_{1}, \ldots, T_{N}\right\}$ is the hyperperiod;

7. $\eta_{i}\left(t_{1}, t_{2}\right)=\left(\left\lfloor\frac{t_{2}-\phi_{i}-D_{i}}{T_{i}}\right\rfloor-\left\lceil\frac{t_{1}-\phi_{i}}{T_{i}}\right\rceil+1\right)_{0}$ is the number of jobs of task $\tau_{i}$ with release time greater than or equal to $t_{1}$ and deadline less then or equal to $t_{2}$ (Baruah et al., 1990b).

Notation $(x)_{0}$ is an abbreviation of $\max (x, 0)$.

A schedule is a function $\sigma: \mathbb{N} \rightarrow \mathcal{T} \cup\{\emptyset\}$ that assigns to each time slot $t$ a task $\tau_{i}$, or the symbol $\varnothing$ to indicate that the processor is idle. A busy period $\left[t_{1}, t_{2}\right)$ is an interval of time in which the processor is always busy.

Synchronization among tasks is carried out through critical sections of code, that uses shared resources. The usage of critical sections ensures that all resources are accessed in exclusive mode. To simplify our presentation, only single-unit resources are considered, although there are ways to consider the case of multi-unit resources (Baker, 1991). We consider a set $\mathcal{R}$ of $\mathrm{R}$ shared resources $\rho_{1}, \ldots, \rho_{R}$. Each task $\tau_{i}$ may access $N_{i}$ different critical sections. Each critical section $\xi_{i j}$ is described by a 3 -ple $\left(\rho_{i j}, \psi_{i j}, C_{i j}\right)$, where:

1. $\rho_{i j} \in \mathcal{R}$ is the resource being accessed;

2. $\psi_{i j}$ is the earliest time, relative to the release time of job $\tau_{i j}$, that the task can enter $\xi_{i j}$;

3. $C_{i j}$ is the worst-case computation time of the critical section.

Critical sections can be properly nested in any arbitrary way, as long as their earliest entry time and worst-case computation time is known. Note that our model, first proposed in Lipari and Buttazzo (2000), is actually slightly different from the classic one used in the 
literature in that it requires earliest entry times to be known. Note that if earliest entry times are unknow, they can simply be set to zero, although this will lead to increased pessimism in the analysis.

In all the figures showing a schedule, we represent the execution of each task on a separate horizontal line. Upward arrows represent release times while downward arrows represent absolute deadlines.

\section{Feasibility Analysis}

In this section, we will first show the fundamental results for the problem of feasibility analysis of periodic task sets on single processor systems. Then we present our idea and prove it correct. Throughout this section we suppose that tasks are independent, i.e. no resource other than the processor is shared among tasks. An extension to the case of shared resources will be presented in Section 4.

Our analysis is based on the processor demand criterion (Baruah et al., 1990b; Buttazzo, 1997). The processor demand function is defined as

$$
d f\left(t_{1}, t_{2}\right)=\sum_{i=1}^{N} \eta_{i}\left(t_{1}, t_{2}\right) C_{i}
$$

It is the amount of time demanded by the tasks in interval $\left[t_{1}, t_{2}\right)$ that the processor must execute to ensure that no task misses its deadline. Intuitively, the following is a necessary condition for feasibility:

$$
\forall 0 \leq t_{1}<t_{2}: d f\left(t_{1}, t_{2}\right) \leq t_{2}-t_{1}
$$

In plain words, the amount of time demanded by the task set in any interval must never be larger than the length of the interval.

\subsection{Existing Results on Feasibility Analysis}

We report two fundamental results on the schedulability analysis of a periodic task set with EDF. The proofs of these results are different from the original ones. They have been rewritten to clarify our methodology.

Lemma 1 (Baruah et al., 1990b). Task set $\mathcal{T}$ is feasible on a single processor if and only if:

1. $U \leq 1$, and

2. $\forall 0 \leq t_{1}<t_{2} \leq \Phi+2 H: d f\left(t_{1}, t_{2}\right) \leq t_{2}-t_{1}$.

Proof: Both conditions are clearly necessary. By contradiction. Suppose both conditions hold but $\mathcal{T}$ is not feasible. Consider the schedule generated by EDF. It can be proven (Baruah et al., 1990b; Leung and Merril, 1980) that since $U \leq 1$ and the task set is not feasible, some 


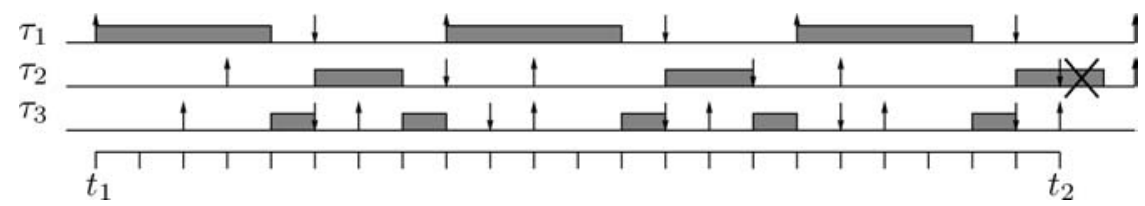

Figure 1. Example of busy period.

deadline in $(0, \Phi+2 H]$ is missed. Let $t_{2}$ be the first instant at which a deadline is missed, and let $t_{1}$ be the last instant prior to $t_{2}$ such that either no jobs or a job with deadline greater than $t_{2}$ is scheduled at $t_{1}-1$. By choice of $t_{1}$, it follows that $\left[t_{1}, t_{2}\right)$ is a busy period and all jobs that are scheduled in $\left[t_{1}, t_{2}\right)$ have arrival times and deadlines in $\left[t_{1}, t_{2}\right]$ (see Figure 1 ). Also, at least one job with deadline no later than $t_{2}$ is released exactly at $t_{1}$, otherwise either a job with deadline greater than $t_{2}$ or no job would be scheduled at $t_{1}$. Since there is no idle time in $\left[t_{1}, t_{2}\right)$ and the deadline at $t_{2}$ is missed, the amount of work to be done in $\left[t_{1}, t_{2}\right)$ exceeds the length of the interval. By definition of $d f$, it follows that $d f\left(t_{1}, t_{2}\right)>t_{2}-t_{1}$, which contradicts condition 2 .

By looking at the proof, it follows that it is sufficient to check the values of $d f\left(t_{1}, t_{2}\right)$ for all times $t_{1}$ that correspond to the release time of some job. In the same way, we can check only those $t_{2}$ that correspond to the absolute deadline of some job.

We will now prove in the following theorem that for a synchronous task set the first deadline miss, if any, is found in the longest busy period starting from $t_{1}=0$ (usually referred to as the critical instant). Thus, it suffices to check all deadlines from 0 to the first idle time. The idea is that, given any busy period starting at $t_{1}$, we can always produce a "worst-case" busy period by "pulling back" the release times of all tasks that are not released at $t_{1}$, so that all tasks are released at the same time. Figures 2 and 3 shows the idea for a task set of $N=3$ tasks with $\tau_{1}(C=4, D=6, T=10), \tau_{2}(C=4, D=12, T=12), \tau_{3}(C=$ $4, D=10, T=14)$. Figure 2 shows a busy period starting at $t_{1}$ with the release of task $\tau_{1}$, while tasks $\tau_{2}$ and $\tau_{3}$ are released at $t_{1}+2$ and $t_{1}+3$ respectively; task $\tau_{3}$ misses its deadline at $t_{2}=t_{1}+27$. Figure 3 shows the busy period obtained by "pulling back" $\tau_{2}$ and $\tau_{3}$ until their release times coincide with that of $\tau_{1}$. Note that the newly obtained busy period

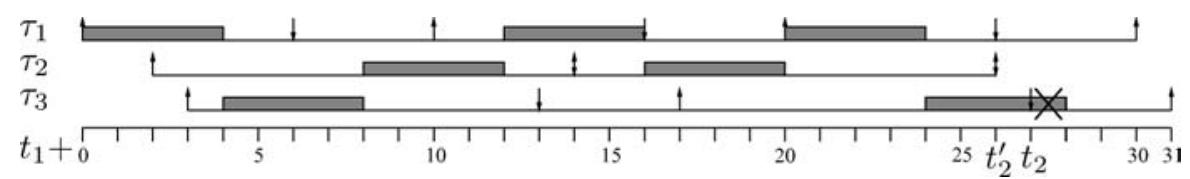

Figure 2. Example: asynchronous task set.

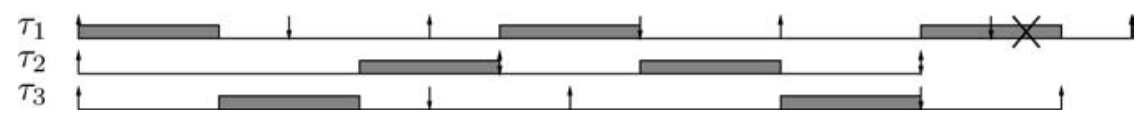

Figure 3. Example: corresponding synchronous task set. 
corresponds to the synchronous busy period starting at 0 , and that while the second job of $\tau_{3}$ now finishes at its deadline, there is still a task, in this case $\tau_{1}$, that misses its deadline at $t_{2}^{\prime}=t_{1}+26$.

Theorem 1 (Baruah et al., 1990b). A synchronous task set $\mathcal{T}$ is feasible on a single processor if and only if:

$$
\forall L \leq L^{\star}, \quad d f(0, L) \leq L
$$

where $L$ is an absolute deadline and $L^{\star}$ is the first idle time in the schedule.

Proof: The condition is clearly necessary, so it remains to prove the sufficiency by contradiction. Consider the schedule generated by EDF. Suppose that a deadline is missed, and let $\left[t_{1}, t_{2}\right)$ be a busy period as in the previous lemma. We already proved that there is at least one task that is released exactly at $t_{1}$. Let $\tau_{i}$ be one such task, so that $t_{1}=r_{i m}$ for some $m$, and $\tau_{k}$ be the task whose deadline $d_{k p}$ is not met (note that it could be $i=k$ ). By following the same reasoning as in Lemma 1 , we obtain $d f\left(t_{1}, t_{2}\right)>t_{2}-t_{1}$.

Now consider a task $\tau_{j}, j \neq i, k$, and let $r_{j l}$ be the first release time of a job of $\tau_{j}$ such that $r_{j l}>t_{1}$. The new schedule generated by "pulling back" all releases of task $\tau_{j}$ of $r_{j l}-t_{1}$ is still unfeasible. In fact, since all absolute deadlines of task $\tau_{j}$ are now located earlier, the number of jobs of $\tau_{j}$ in $\left[t_{1}, t_{2}\right]$ could be increased: $\eta_{j}^{\prime}\left(t_{1}, t_{2}\right) \geq \eta_{j}\left(t_{1}, t_{2}\right)$. Thus, $\sum_{i=1, i \neq j}^{N} \eta_{i}\left(t_{1}, t_{2}\right) C_{i}+\eta_{j}^{\prime}\left(t_{1}, t_{2}\right) C_{j} \geq \sum_{i=1}^{N} \eta_{i}\left(t_{1}, t_{2}\right) C_{i}>t_{2}-t_{1}$ (see task $\tau_{2}$ in Figures 2 and 3). Now consider task $\tau_{k}$, and suppose that $k \neq i$. Let $r_{k l}$ be the first release time of $\tau_{k}$ after $t_{1}$. By moving all releases back of $r_{k l}-t_{1}$, we also move back its deadlines. Let $d_{k p}^{\prime} \leq d_{k p}$ be the new deadline. We shall consider two possible cases. First, suppose that for each deadline $d_{j q} \leq d_{k p}, j \neq k$, it still holds $d_{j q} \leq d_{k p}^{\prime}$. Then $d f\left(t_{1}, t_{2}^{\prime}=d_{k p}^{\prime}\right)=$ $d f\left(t_{1}, t_{2}\right)>t_{2}-t_{1}>t_{2}^{\prime}-t_{1}$ and the new task set is not feasible. Second, suppose that $d_{j q}$ is the largest deadline in $\left[t_{1}, t_{2}\right]$ such that $d_{k p}^{\prime}<d_{j q} \leq d_{k p}$. Consider the new busy period $\left[t_{1}, t_{2}^{\prime}=d_{j q}\right)$. Then $d f\left(t_{1}, t_{2}^{\prime}\right)=d f\left(t_{1}, t_{2}\right)$, with $t_{2}^{\prime} \leq t_{2}$, thus the new task set is not feasible (see Figures 2 and 3, where $k=3$ and $j=1$ ).

Therefore, by moving back all tasks such that their first release time is at $t_{1}$ we obtain an unfeasible schedule where all tasks are simultaneously released at $t_{1}$. Thus, if a deadline is missed inside any busy period, then a deadline is missed inside the busy period starting at 0 . Moreover, $d f\left(0, t_{2}-t_{1}\right) \geq d f\left(t_{1}, t_{2}\right)>t_{2}-t_{1}$. This contradicts the hypothesis, hence the theorem holds.

It can be proved that for $U<1$ the busy period length is bounded by $\frac{U}{1-U} \max _{i=1}^{N}\left\{T_{i}-D_{i}\right\}$ (Baruah et al., 1990b). Therefore, the analysis has complexity $O\left(N \frac{U}{1-U} \max _{i=1}^{N}\left\{T_{i}-D_{i}\right\}\right)$.

The previous theorem does not hold in the case of asynchronous task sets. It still gives a sufficient condition, in the sense that if the hypothesis holds for the corresponding synchronous task set, than the original asynchronous task set is feasible. However the condition is no longer necessary. Consider the feasible task set in Figure 4, composed of tasks $\tau_{1}(\phi=1, C=2, D=3, T=4)$ and $\tau_{2}(\phi=0, C=2, D=3, T=6)$. Since we are showing the schedule in a full hyperperiod, it should be easy to see that no instant $t_{1}$ exists such that both tasks are released simultaneously. We can still use a pessimistic analysis 


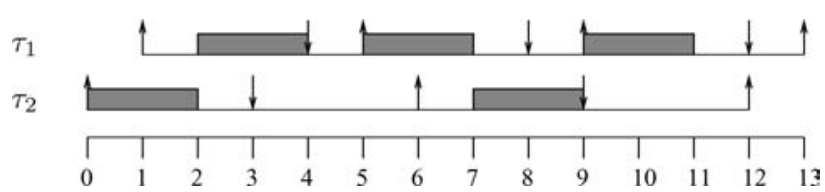

Figure 4. Example task set.

by considering the corresponding synchronous task set, but in the case of Figure 4 this would not work since it can be easily seen that the corresponding synchronous task set is not feasible; in fact, if we move back the deadline of the first job of $\tau_{1}$ to 3 , we obtain $d f(0,3)=4>3$ and therefore a deadline will be missed. Checking all busy periods in $[0, \Phi+2 H]$ is possible but would imply an exponential complexity.

\subsection{Improving the Analysis for Asynchronous Tasks}

The intuition behind our idea is as follow. In the proofs of Lemma 1 and Theorem 1 we note that there is always an initial task that is released at $t_{1}$, the start of the "critical" busy period. However, we do not know which task is it. Therefore, we build a new task set $\mathcal{T}_{i}^{\prime}$ for each possible initial task $\tau_{i}, 1 \leq i \leq N$. Since $\tau_{i}$ is released at the beginning of the busy period, we fix $\phi_{i}^{\prime}=0$ in $\mathcal{T}_{i}^{\prime}$ and check the busy period starting from 0 instead of $t_{1}$. We can then "pull back" each other task $\tau_{j}$ by setting $\phi_{j}^{\prime}$ to the minimum time distance between any activation of $\tau_{i}$ and the successive activation of $\tau_{j}$ in the original task set $\mathcal{T}$. We will use the following Lemma:

Lemma 2. Given two tasks $\tau_{i}$ and $\tau_{j}$, the minimum time distance between any release time of task $\tau_{i}$ and the successive release time of task $\tau_{j}$ is equal to:

$$
\Delta_{i j}=\phi_{j}-\phi_{i}+\left\lceil\frac{\phi_{i}-\phi_{j}}{\operatorname{gcd}\left(T_{j}, T_{i}\right)}\right\rceil \operatorname{gcd}\left(T_{j}, T_{i}\right)
$$

Proof: Note that for each possible job $\tau_{i m}$ and $\tau_{j l}, r_{j l}-r_{i m}=\phi_{j}-\phi_{i}+l T_{j}-m T_{i}$. Thus $\forall l \geq 0, \forall m \geq 0, \exists K \in \mathbb{Z}, r_{j l}-r_{i m}=\phi_{j}-\phi_{i}+K \operatorname{gcd}\left(T_{j}, T_{i}\right)$. By imposing $r_{j l} \geq r_{i m}$, we obtain $K \geq\left\lceil\frac{\phi_{i}-\phi_{j}}{\operatorname{gcd}\left(T_{j}, T_{i}\right)}\right\rceil$. By simple substitution, we obtain the lemma.

Definition 1. Given task set $\mathcal{T}, \mathcal{T}_{i}^{\prime}$ is the task set with the same tasks as $\mathcal{T}$ but with offsets:

$$
\begin{aligned}
& \phi_{i}^{\prime}=0 \\
& \phi_{j}^{\prime}=\Delta_{i j} \quad \forall j \neq i, 1 \leq j \leq N
\end{aligned}
$$

Consider the example of Figure 5. By setting $i=1$ we obtain $\phi_{1}^{\prime}=0, \phi_{2}^{\prime}=r_{23}-r_{14}=0$ and $\phi_{3}^{\prime}=r_{31}-r_{11}=2$. 


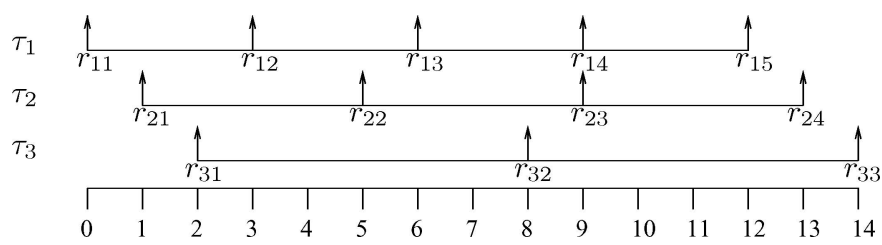

Figure 5. Example task set, $\tau_{1}(\phi=0, T=3), \tau_{2}(\phi=1, T=4), \tau_{3}(\phi=2, T=6)$.

We will now prove that, to assess the feasibility of $\mathcal{T}$, it suffices to check that, for every task set $\mathcal{T}_{i}^{\prime}, 1 \leq i \leq N$, all deadlines are met inside the busy period starting from time 0 .

Theorem 2. Given task set $\mathcal{T}$ with $U \leq 1$, scheduled on a single processor, if $\forall 1 \leq i \leq$ $N$ all deadlines in task set $\mathcal{T}_{i}^{\prime}$ are met until the first idle time, then $\mathcal{T}$ is feasible.

Proof: By contradiction. Consider the schedule generated by EDF. Suppose that a deadline is not met for task set $\mathcal{T}$, and let $\left[t_{1}, t_{2}\right)$ be the busy period as defined in Lemma 1 . We already proved that there is at least one task that is released at $t_{1}$, let it be $\tau_{i}$. From Lemma 2, it follows that for every $\tau_{j}, j \neq i$, the successive release time is $r_{j l} \geq t_{1}+\Delta_{i j}$. By following the same reasoning as in Theorem 1, we can "pull back" every task so that its first release time coincides with its minimum distance from $t_{1}$, and the resulting schedule is still unfeasible. Let $\sigma_{i}(t)$ be the new resulting schedule.

Now, observe that, from $t_{1}$ on, the new schedule $\sigma_{i}(t)$ is coincident with the schedule $\sigma_{i}^{\prime}(t)$ generated by task set $\mathcal{T}_{i}^{\prime}$ from time $0: \forall t \geq t_{1}: \sigma_{i}(t)=\sigma_{i}^{\prime}\left(t-t_{1}\right)$. Therefore, there is a deadline miss in the first busy period in the schedule generated by $\mathcal{T}_{i}^{\prime}$, against the hypothesis. Hence, the theorem follows.

Note that Theorem 2 gives us a less pessimistic feasibility condition that Theorem 1. As an example, consider the task set in Figure 4. According to Theorem 2 the task set is feasible (the minimum distance between either $\tau_{1}$ and $\tau_{2}$ or viceversa is equal to 1 , therefore there is always idle time at 4 ), while Theorem 1 gives no result.

However, Theorem 2 gives only a sufficient condition. For example, consider the following task set: $\tau_{1}(\phi=0, C=1, D=2, T=5), \tau_{2}(\phi=1, C=1, D=2, T=4), \tau_{3}(\phi=$ 2, $C=1, D=2, T=6$ ). By analyzing the schedule, it can be seen that it is feasible, but Theorem 2 fails to give any result. The reason can be easily explained. When we "pull back" the tasks to their minimum distance from $\tau_{i}$, we are not considering the cross relations between them. In other words, it may be possible that the pattern of release times analyzed with Theorem 2 are not found in the original schedule of $\mathcal{T}$.

\subsection{Generalization}

In order to reduce the pessimism in the analysis, we can generalize Theorem 2 in the following way. Instead of fixing just the initial task $\tau_{i}$, we can also fix the position of other tasks with respect to $\tau_{i}$ and then minimize the offsets of all the remaining tasks with respect to the fixed ones. The following lemma provides deeper insight. 
Lemma 3. The time distance between any release time $r_{i l}$ of task $\tau_{i}$ and the successive release time $r_{j p}$ of task $\tau_{j}$ assumes values inside the following set:

$$
\left\{\Delta_{i j}(k) \mid \forall 0 \leq k<\frac{T_{j}}{\operatorname{gcd}\left(T_{i}, T_{j}\right)}\right\}
$$

where

$$
\Delta_{i j}(k)=\left\lceil\frac{\phi_{i}+k T_{i}-\phi_{j}}{T_{j}}\right\rceil T_{j}-\left(\phi_{i}+k T_{i}-\phi_{j}\right)
$$

Proof: Consider release time $r_{i l}$ and let $r_{j p}$ be the first successive release time of task $\tau_{j}$. Since $r_{j p}$ is greater than or equal to $r_{i l}$, it must be $\phi_{j}+p T_{j} \geq \phi_{i}+l T_{i}$. But since $r_{j p}$ is the first such release, we obtain $p=\left\lceil\frac{\phi_{i}+l T_{i}-\phi_{j}}{T_{j}}\right\rceil$ and $r_{j p}-r_{i l}=\left\lceil\frac{\phi_{i}+l T_{i}-\phi_{j}}{T_{i}}\right\rceil T_{j}-\left(\phi_{i}+l T_{i}-\phi_{j}\right)$. To end the proof it suffices to note that the value of $r_{j p}-r_{i l}$ as a function of $l$ is periodic of period $\frac{T_{j}}{\operatorname{gcd}\left(T_{i}, T_{j}\right)}$. In fact:

$$
\begin{aligned}
& {\left[\frac{\phi_{i}+\left(l+K \frac{T_{i}}{\operatorname{gcd}\left(T_{i}, T_{j}\right)}\right) T_{i}-\phi_{j}}{T_{j}}\right] T_{j}-\left(\phi_{i}+\left(l+K \frac{T_{j}}{\operatorname{gcd}\left(T_{i}, T_{j}\right)}\right) T_{i}\right)+\phi_{j}} \\
& \quad=\left\lceil\frac{\phi_{i}+l T_{+} K \frac{T_{j} T_{i}}{\operatorname{gcd}\left(T_{i}, T_{j}\right)}-\phi_{j}}{T_{j}}\right] T_{j}-\left(\phi_{i}+l T_{i}+K \frac{T_{j} T_{i}}{\operatorname{gcd}\left(T_{i}, T_{j}\right)}\right)+\phi_{j} \\
& \quad=r_{j p}-r_{i l}
\end{aligned}
$$

Therefore, after fixing the first task $\tau_{i}$ we can fix another task $\tau_{j}$ to one of the values of Lemma 3. Now, if we want to fix a third task, we must be careful to select a time instant that is compatible with the release times of both $\tau_{i}$ and $\tau_{j}$. The basic idea, explained in the following lemma, is to consider $\tau_{i}$ and $\tau_{j}$ as a single task of period $\operatorname{lcm}\left(T_{i}, T_{j}\right)$ and offset $\phi_{i}+k T_{i}$.

To generalize the notation, we denote with $i_{1}$ the index of the first task that is fixed, with $i_{2}$ the index of the second task, and so on, until $i_{M}$ that denotes the index of the last task to be fixed.

Lemma 4. Let $r_{i_{1} l_{1}}$ be any release time of task $\tau_{i_{1}}$, and let $\Delta_{i_{1} i_{2}}\left(k_{1}\right)$ be the distance between $r_{i_{1} l_{1}}$ and the successive release time of task $\tau_{i_{2}}$. The time distance between $r_{i_{1} l_{1}}$ and the successive release time of task $\tau_{i_{3}}$ assumes values inside the following set:

$$
\left\{\Delta_{i_{1} i_{2} i_{3}}\left(k_{2}\right) \mid \forall 0 \leq k_{2}<\frac{T_{i_{3}}}{\operatorname{gcd}\left(T_{i_{3}}, \operatorname{lcm}\left(T_{i_{1}}, T_{i_{2}}\right)\right)}\right\}
$$


where

$$
\begin{aligned}
\Delta_{i_{1} i_{2} i_{3}}\left(k_{2}\right)= & \left\lceil\frac{\phi_{i_{1}}+k_{1} T_{i_{1}}+k_{2} \operatorname{lcm}\left(T_{i_{1}}, T_{i_{2}}\right)-\phi_{i_{3}}}{T_{i_{3}}}\right\rceil T_{i_{3}}+ \\
& -\left(\phi_{i_{1}}+k_{1} T_{i_{1}}+k_{2} \operatorname{lcm}\left(T_{i_{1}}, T_{i_{2}}\right)-\phi_{i_{3}}\right)
\end{aligned}
$$

Proof: Since the time difference between $r_{i_{1} l_{1}}$ and the successive release time $r_{i_{2} l_{2}}$ of $\tau_{i_{2}}$ must be equal to $\Delta_{i_{1} i_{2}}\left(k_{1}\right)$, not all values of $l_{1}$ are acceptable. Indeed, it must hold $l_{1} \equiv k_{1} \bmod \left(\frac{T_{i_{2}}}{\operatorname{gcd}\left(T_{i_{1}}, T_{i_{2}}\right)}\right)$.

Let $\tau_{i_{1} i_{2}}$ be a task with period $T_{i_{1} i_{2}}=T_{i_{1}} \frac{T_{i_{2}}}{\operatorname{gcd}\left(T_{i_{1}}, T_{i_{2}}\right)}=\operatorname{lcm}\left(T_{i_{1}}, T_{i_{2}}\right)$ and offset $\phi_{i_{1} i_{2}}=$ $\phi_{i_{1}}+k_{1} T_{i_{1}}$. All acceptable release times $r_{i_{1} l_{1}}$ correspond to the release times of task $\tau_{i_{1} i_{2}}$. We can then apply Lemma 3 to $\tau_{i_{1} i_{2}}$ and $\tau_{i_{3}}$ obtaining $\Delta_{i_{1} i_{2} i_{3}}$.

Lemma 5. The time distance between any release time $r_{i_{1} l_{1}}$ of task $\tau_{i_{1}}$ and the successive release time of task $\tau_{i_{p}}$, given $r_{i_{2} l_{2}}-r_{i_{1} l_{1}}=\Delta_{i_{1} i_{2}}\left(k_{1}\right), \ldots, r_{i_{p-1} l_{p-1}}-r_{i_{1} l_{1}}=\Delta_{i_{1} \ldots i_{p-1}}\left(k_{p-2}\right)$, assumes values inside the following set:

$$
\left\{\Delta_{i_{1} \ldots i_{p}}\left(k_{p-1}\right) \mid \forall 0 \leq k_{p-1}<\frac{T_{i_{p}}}{\operatorname{gcd}\left(T_{i_{p}}, \operatorname{lcm}\left(T_{i_{1}}, \ldots, T_{i_{p-1}}\right)\right)}\right\}
$$

where

$$
\begin{aligned}
\Delta_{i_{1} \ldots i_{p}}\left(k_{p-1}\right)= & \left\lceil\frac{\phi_{i_{1}}+\sum_{q=1}^{p-1} k_{q} \operatorname{lcm}\left(T_{i_{1}}, \ldots, T_{i_{q}}\right)-\phi_{i_{p}}}{T_{i_{p}}}\right] T_{i_{p}}+ \\
& -\left(\phi_{i_{1}}+\sum_{q=1}^{p-1} k_{q} \operatorname{lcm}\left(T_{i_{1}}, \ldots, T_{i_{q}}\right)-\phi_{i_{p}}\right)
\end{aligned}
$$

Proof: The proof can be obtained by induction, reasoning in the same way as in Lemma 4 .

Lemma 6. The minimum time distance between any release time $r_{i_{1} l_{1}}$ of task $\tau_{i_{1}}$ and the successive release time of task $\tau_{j}$, given $r_{i_{2} l_{2}}-r_{i_{1} l_{1}}=\Delta_{i_{1} i_{2}}\left(k_{1}\right), \ldots, r_{i_{M} l_{M}}-r_{i_{1} l_{1}}=$ $\Delta_{i_{1} \ldots i_{M}}\left(k_{M-1}\right)$, is equal to:

$$
\begin{aligned}
\Delta_{i_{1} \ldots i_{M} j}= & \phi_{j}-\phi_{i_{1}}-\sum_{q=1}^{M-1} k_{q} \operatorname{lcm}\left(T_{i_{1}}, \ldots, T_{i_{q}}\right) \\
& +\left\lceil\frac{\phi_{i_{1}}+\sum_{q=1}^{M-1} k_{q} \operatorname{lcm}\left(T_{i_{1}}, \ldots, T_{i_{q}}\right)-\phi_{j}}{\operatorname{gcd}\left(T_{j}, \operatorname{lcm}\left(T_{i_{1}}, \ldots, T_{i_{M}}\right)\right)}\right\rceil \cdot \operatorname{gcd}\left(T_{j}, \operatorname{lcm}\left(T_{i_{1}}, \ldots, T_{i_{M}}\right)\right)
\end{aligned}
$$


Proof: Reasoning in the same way as in Lemmas 4 and 5, all acceptable release times $r_{i_{1}} l_{1}$ must correspond to the release times of a task $\tau_{i_{1} \ldots i_{M}}$ with period $T_{i_{1} \ldots i_{M}}=\operatorname{lcm}\left(T_{i_{1}}, \ldots, T_{i_{M}}\right)$ and offset $\phi_{i_{1} \ldots i_{M}}=\phi_{i_{1}}+\sum_{q=1}^{M-1} k_{q} \operatorname{lcm}\left(T_{i_{1}}, \ldots, T_{i_{q}}\right)$. We can then apply Lemma 2 to $\tau_{i_{1} \ldots i_{M}}$ and $\tau_{j}$ obtaining $\Delta_{i_{1} \ldots i_{M} j}$.

Following the same line of reasoning as in Theorem 2, we now define task set $\mathcal{T}_{i_{1} \ldots i_{M} k_{1} \ldots k_{M-1}}^{\prime}$ in the same way as $\mathcal{T}_{i}^{\prime}$ before.

Definition 2. Given task set $\mathcal{T}, \mathcal{T}_{i_{1} \ldots i_{M} k_{1} \ldots k_{M-1}}^{\prime}$ is the task set with the same tasks as $\mathcal{T}$ but with offsets:

$$
\begin{aligned}
\phi_{i_{1}}^{\prime} & =0 \\
\vdots & \vdots \\
\phi_{i_{p}}^{\prime} & =\Delta_{i_{1} \ldots i_{p}}\left(k_{p-1}\right) \\
\vdots & \vdots \\
\phi_{i_{M}}^{\prime} & =\Delta_{i_{1} \ldots i_{M}}\left(k_{M-1}\right) \\
\phi_{j}^{\prime} & =\Delta_{i_{1} \ldots i_{M} j} \quad \forall j \neq i_{1}, \ldots, i_{M}, 1 \leq t \leq N
\end{aligned}
$$

Finally, we generalize Theorem 2 to the case of $M$ fixed tasks.

Theorem 3. Given task set $\mathcal{T}$ with $U \leq 1$, to be scheduled on a single processor, let $M$ be a number of tasks, $1 \leq M<N$. If:

$$
\begin{aligned}
& \forall \tau_{i_{1}}, 1 \leq i_{1} \leq N, \forall \tau_{i_{2}} \neq \tau_{i_{1}}, 1 \leq i_{2} \leq N, \ldots, \\
& \forall \tau_{i_{M}} \neq \tau_{i_{1}}, \tau_{i_{2}}, \ldots, \tau_{i_{M-1}}, 1 \leq i_{M} \leq N \\
& \forall k_{1}, 0 \leq k_{1}<\frac{T_{i_{2}}}{\operatorname{gcd}\left(T_{i_{1}}, T_{i_{2}}\right)}, \forall k_{2}, 0 \leq k_{2}<\frac{T_{i_{3}}}{\operatorname{gcd}\left(T_{i_{3}}, \operatorname{lcm}\left(T_{i_{1}}, T_{i_{2}}\right)\right)}, \ldots, \\
& \forall k_{M-1}, 0 \leq k_{M-1}<\frac{T_{i_{M}}}{\operatorname{gcd}\left(T_{i_{M}}, \operatorname{lcm}\left(T_{i_{1}}, \ldots, T_{i_{M-1}}\right)\right)},
\end{aligned}
$$

all deadlines in task set $\mathcal{T}_{i_{1} \ldots i_{M} k_{1} \ldots k_{M-1}}^{\prime}$ are met until the first idle time, then $\mathcal{T}$ is feasible.

Proof: By contradiction. Consider the schedule generated by EDF. Suppose that a deadline is missed and let $\left[t_{1}, t_{2}\right)$ be the busy period as in the proof of Lemma 1 . We choose $i_{1}$ such that $t_{1}=r_{i_{1} l_{1}}$ for some $l_{1}$. Next, we choose any distinct indexes $i_{2}, \ldots, i_{M}$ and any values $k_{1}, \ldots, k_{M-1}$ as in Lemmas 3, 4, 5, and compute the corresponding distances $\Delta_{i_{1} i_{2}}\left(k_{1}\right), \ldots, \Delta_{i_{1} \ldots i_{M}}\left(k_{M-1}\right)$ from $t_{1}$. These tasks are fixed and will not be "pulled back". For the remaining tasks, we "pull back" their release times as much as it is possible: for every non-fixed task $\tau_{j}$ we set the distance from $t_{1}$ equal to $\Delta_{i_{1} \ldots i_{M} j}$. By following the same reasoning as in Theorem 2 , it can be easily proven that a deadline is still missed in the new 
generated schedule $\sigma(t)$. Note that, from $t_{1}$ on, the schedule $\sigma(t)$ is coincident with the schedule $\sigma^{\prime}(t)$ generated by task set $\mathcal{T}_{i_{1} \ldots i_{M} k_{1} \ldots k_{M-1}}^{\prime}$ from time $0: \forall t \geq t_{1}: \sigma(t)=\sigma^{\prime}\left(t-t_{1}\right)$. Hence, a deadline is missed in the first busy period of $\sigma^{\prime}(t)$, against the hypothesis.

\subsection{Analysis of Mixed Sporadic and Periodic Task Sets}

In the previous section we considered task sets consisting only of periodic tasks. Although many real-time activities can be modelled by periodic tasks, there are many others that are usually modelled by using sporadic tasks, i.e. aperiodic tasks with a minimum inter-arrival time. Therefore, we now extend our analysis to the case of sporadic tasks.

First note that the concept of offset is meaningless for a sporadic task, since it does not follow a periodic activation pattern; a sporadic job can be activated anywhere in the schedule at the same time as any other task. Therefore, our asynchronous analysis does not yield any result for task set composed of sporadic tasks only. We are instead interested in analyzing task sets composed of both periodic and sporadic tasks.

We will consider a mixed periodic-sporadic task set $\mathcal{T}$ composed of $N$ periodic tasks $\tau_{1}, \ldots, \tau_{N}$ and $M$ sporadic tasks $\tau_{N+1}, \ldots, \tau_{N+M}$. Each task is defined by the same parameters as in Section 2, except that for a sporadic task $\tau_{i}, T_{i}$ represents its minimum inter-arrival time and offset $\phi_{i}$ is meaningless.

We can easily extend out test with one fixed task from Theorem 2 to mixed periodsporadic task sets, simply considering that the minimum distance between the activation of a sporadic task and the successive activation of any other task is always zero. The extension is more formally defined as follows.

Definition 3. Given a mixed periodic-sporadic task set $\mathcal{T}, \mathcal{T}_{i}^{\prime}$ is the task set with the same tasks as $\mathcal{T}$ but with offsets:

$$
\begin{aligned}
\phi_{i}^{\prime} & =0 \\
\phi_{j}^{\prime} & =\Delta_{i j} \quad \forall j \neq i, 1 \leq j \leq N \\
\phi_{j}^{\prime} & =0 \quad \forall j, N<j \leq N+M
\end{aligned}
$$

Theorem 4. Given a mixed periodic-sporadic task set $\mathcal{T}$ with $U \leq 1$, scheduled on a single processor, if $\forall 1 \leq i \leq N$ all deadlines in task set $\mathcal{T}_{i}^{\prime}$ are met until the first idle time, then $\mathcal{T}$ is feasible.

Proof: By contradiction. Suppose that a deadline is not met for task set $\mathcal{T}$, and let $\left[t_{1}, t_{2}\right)$ be the busy period as defined in Lemma 1 . We must consider two cases. First, suppose that a periodic task $\tau_{i}$ is released at $t_{1}$. We can then follow the same proof as in Theorem 2 , "pulling back" all sporadic tasks so that their first release time coincides with $t_{1}$.

If no periodic task is released at $t_{1}$, than a sporadic task $\tau_{j}$ must surely be released at $t_{1}$. Let $\tau_{i}$ be the periodic task with the smallest activation time (suppose $r_{i k}$ ) inside the busy period. If we pull back all periodic tasks of an amount of time units equal to $r_{i k}-t_{1}$, the resulting schedule is still unfeasible as proven in Theorem 1 . Let $\sigma_{i}(t)$ be the new resulting 
schedule after pulling back all other sporadic tasks so that their first release time coincides with $t_{1}$. The new schedule $\sigma_{i}(t)$ is coincident with the schedule $\sigma_{i}^{\prime}(t)$ generated by task set $\mathcal{T}_{i}^{\prime}$ from time $0: \forall t \geq t_{1}: \sigma_{i}(t)=\sigma_{i}^{\prime}\left(t-t_{1}\right)$; therefore, there is a deadline miss in the first busy period in the schedule generated by $\mathcal{T}_{i}^{\prime}$, against the hypothesis. Hence, the theorem follows.

It is possible to extend Theorem 3 in the same straightforward way; the extension is not shown due to space constraint.

\subsection{Algorithms}

Theorem 2 gives us a new feasibility test for asynchronous task sets with $U<1$ on single processor systems. For each initial task $\tau_{i}$ we first compute the minimal offset $\phi_{j}$ for each $j \neq i$ and the length $L^{*}$ of the busy period. Then, we check that each deadline $L$ less than or equal to $L^{*}$ is met. The pseudo code is given in Figure 6.

Note that the recurrence over the length of the busy period $L^{*}(t+1)=\sum_{i=1}^{N}\left(\left\lceil\frac{L^{*}(t)-\phi_{i}}{T_{i}}\right\rceil\right)_{0}$ $C_{i}$ converges in pseudo-polynomial time if $U<1$ (Spuri, 1996a).

Since we must execute the algorithm for each initial task $\tau_{i}$, the test has a computational complexity that is $N$ times that of Barauh's synchronous test: $O\left(N^{2} \frac{U}{1-U} \max _{i=1}^{N}\left\{T_{i}-D_{i}\right\}\right)$.

We can obtain a less pessimistic test, at the cost of an increased computation time, by using Theorem 3. As the number of fixed tasks $M$ increases, we can expect to obtain higher percentages of feasible task sets, but the computation complexity rises quickly. If we select $M$ fixed tasks, the complexity is bounded by $O\left(N^{M+1} \max _{i, j=1}^{N}\left\{\frac{T_{i}}{\operatorname{gcd}\left(T_{i}, T_{j}\right)}\right\}^{M-1} \frac{U}{1-U} \max _{i=1}^{N}\left\{T_{i}-D_{i}\right\}\right)$. The pseudo code for $M=2$ is given in Figure 7 .

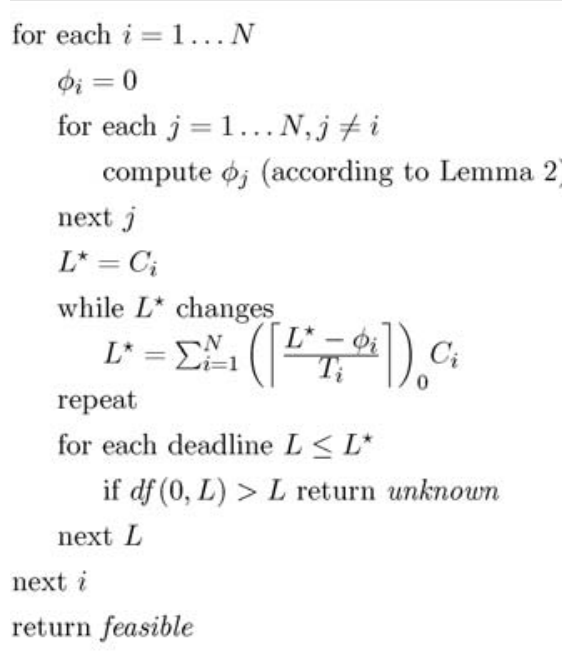

Figure 6. Pseudocode for the feasibility test, 1 fixed task. 


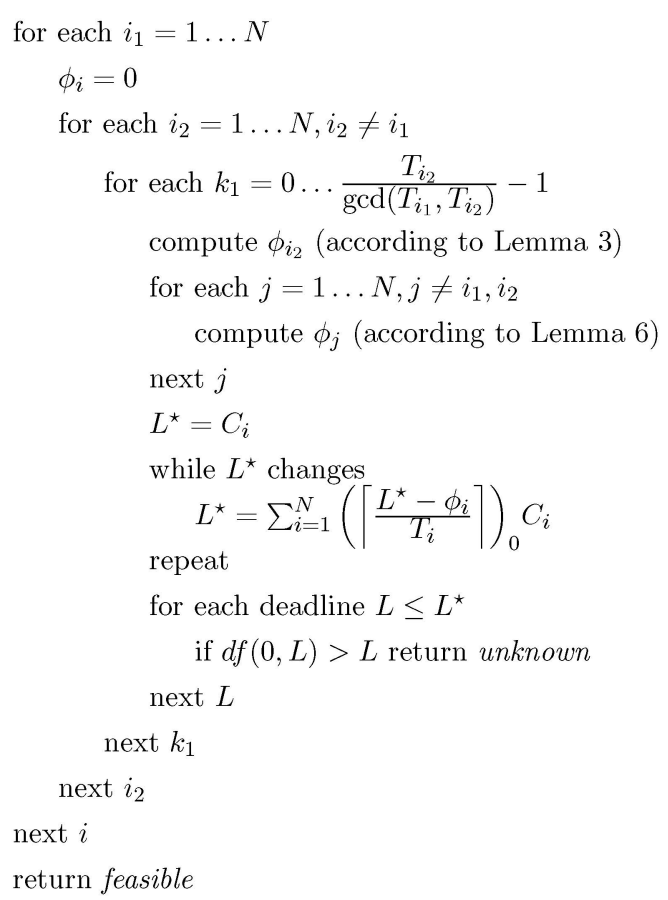

Figure 7. Pseudocode for the feasibility test, 2 fixed tasks.

\section{Taking into Account Shared Resources}

In this section, we will extend the test with 1 fixed task to cover the problem of blocking times and synchronization on shared resources. In order to achieve predictability, a resource access protocol must be introduced in order to bound the maximum blocking time experienced by tasks due to mutual exclusion. Many resource access protocols have been proposed in literature (Chen and Lin, 1990; Jeffay, 1992); we base our discussion on the Stack Resource Protocol (Baker, 1991).

In the remainder of this section, we briefly introduce the SRP and the processor demand criterion with blocking time; our test is a modified version of the one presented in Lipari and Buttazzo (2000). Finally we will extend the analysis in order to account for offsets.

\section{1. $S R P$}

Under SRP, each task is assigned a static preemption level $\pi_{i}=\frac{1}{D_{i}}$ in addition to its dynamic priority defined by EDF. The following fundamental property holds:

Property 1. Task $\tau_{i}$ can preempt task $\tau_{j}$ only if $\pi_{i}>\pi_{j}$. 
To ease further definitions, we will also define an additional preemption level $\pi_{s}$ as a preemption level that is strictly greater than the preemption level of every task.

Furthermore, each resource $\rho_{k}$ is assigned a static ceiling $\operatorname{ceil}\left(\rho_{k}\right)=\max _{i}\left\{\pi_{i} \mid \exists \xi_{i j}, \rho_{i j}=\right.$ $\left.\rho_{k}\right\}$. A dynamic system ceiling is then defined as follows:

$$
\Pi_{s}(t)=\max \left(\left\{\operatorname{ceil}\left(\rho_{k}\right) \mid \rho_{k} \text { is busy at time } t\right\} \cup 0\right)
$$

The scheduling rule is the following: a job is not allowed to start execution until its priority is the highest among the active jobs and its preemption level is strictly higher then the system ceiling.

Among the many useful properties of SRP, we are mainly interested in two of them:

Property 2. ([Baker, 1991]). Under SRP, a job can only be blocked before it starts execution; once started, it can only be preempted by higher priority jobs.

Property 3. ([Baker, 1991]). A job can be blocked only once by one lower priority job.

In the following Section 4.2, we will briefly introduce the processor demand criterion with blocking time; our test is a modified version of the one presented in Lipari and Buttazzo (2000). In Section 4.3 we will then extend the analysis in order to account for offsets.

\subsection{Processor Demand Analysis with Blocking Time}

Since tasks can now share resources, we need to account for blocking times inside busy periods. We will start by proving than no more than one job may cause blocking time inside a busy period:

Lemma 7. Jobs that are completely executed in any busy period $\left[t_{1}, t_{2}\right)$, where $t_{2}$ corresponds to a deadline and $t_{1}$ is the last instant prior to $t_{2}$ such that either no jobs or a job with deadline greater than $t_{2}$ is scheduled at $t_{1}-1$, may be blocked only once by a single lower priority job.

Proof: Without blocking times, all jobs completely executed inside the busy period must be released at or after $t_{1}$ and have deadline at or before $t_{2}$ as in Lemma 1. We will call $\mathcal{A}$ the set of such jobs. However, when blocking times are introduced it is possible for a job of some task $\tau_{j}$ with deadline greater than $t_{2}$ to be executed inside the busy period. For this to be possible, the job must be inside a critical section at time $t_{1}$, since it must block some higher priority job in $\mathcal{A}$. However, there can only be one such job; otherwise, some job in $\mathcal{A}$ would be blocked by at least two lower priority jobs, which is impossible due to Property 3.

Due to Lemma 7, it makes sense to define a dynamic maximum blocking time $B(t)$ as the maximum blocking time that can be experienced by any task inside a busy period of length $t$. 
Definition 4. Given a synchronous task set $\mathcal{T}$, we define the dynamic maximum blocking time for $\mathcal{T}$ as follows:

$$
B(t)=\max _{j k}\left(\left\{C_{j k}-1 \mid D_{j}>\left(t+\psi_{j k}+1\right) \wedge \operatorname{ceil}\left(\rho_{j k}\right) \geq \frac{1}{t}\right\} \cup 0\right)
$$

Lemma 8. $B\left(t_{2}-t_{1}\right)$ is an upper bound to the maximum blocking time experienced by any task completely executed in a busy period $\left[t_{1}, t_{2}\right)$ as defined in Lemma 7.

Proof: As in Lemma 7, let $\mathcal{A}$ be the set of jobs that are released at or after $t_{1}$ and have deadline at or before $t_{2}$. Since jobs in $\mathcal{A}$ can only be blocked by a single lower priority job, the maximum blocking time can be no longer than the length of some critical section $C_{j k}-1$; in fact, the blocking job can enter $\xi_{j k}$ at worst at $t_{1}-1$. Furthermore, since the job cannot enter $\xi_{j k}$ before $\psi_{j k}$ time units have elapsed since its activation, and its deadline must be greater than $t_{2}$, it must also hold $D_{j}>t_{2}-t_{1}+\psi_{j k}+1$. Finally, resource $\rho_{i j}$ must be able to block some job in $\mathcal{A}$; since no job in $\mathcal{A}$ can have a deadline longer than $t_{2}-t_{1}$, it must hold ceil $\left(\rho_{i j}\right) \geq \frac{1}{t}$. Since $B(t)$ considers the critical section of maximum length among the ones that respect the previous conditions, it is surely an upper bound to the maximum blocking time experienced by tasks in $\left[t_{1}, t_{2}\right)$.

Note that the computed bound is clearly pessimistic; in particular, it may be impossible for the blocking job to enter the critical section exactly at $t_{1}-1$.

Once $B(t)$ has been defined, it is trivial to prove the following theorem:

Theorem 5. A synchronous task set $\mathcal{T}$, using mutually exclusive critical sections, is feasible on a single processor if:

$$
\forall L \leq L^{\star}, d f(0, L)+B(L) \leq L
$$

where $L$ is an absolute deadline and $L^{\star}$ is the first idle time in the schedule.

Proof: The theorem is a direct extension of Theorem 1, since tasks executed inside any busy period of length $L$ cannot be blocked for more than $B(L)$ time units as proven in Lemma 8.

\subsection{Extension to Offsets}

We will now extend the analysis to account for asynchronous task sets. When tasks have non zero offsets, we can exploit two different behaviors in order to reduce the computed maximum blocking time. First of all, it may be impossible for the blocking task to be released exactly $\psi_{j k}+1$ time units before the beginning of the busy period. In order to capture this behavior, we need to compute a new minimum time distance between tasks. 
Lemma 9. Given two tasks $\tau_{i}$ and $\tau_{j}$, the minimum time distance between any release time of task $\tau_{i}$ and the successive release time of task $\tau_{j}$ that is greater or equal to some value $q+1$ is equal to:

$$
\Delta_{i j}^{q}=\phi_{j}-\phi_{i}+\left\lceil\frac{\phi_{i}+q+1-\phi_{j}}{\operatorname{gcd}\left(T_{j}, T_{i}\right)}\right\rceil \operatorname{gcd}\left(T_{j}, T_{i}\right)
$$

Proof: The proof is a simple extension of Lemma 2; it is sufficient to see that the condition on $q$ is equivalent to considering $\tau_{i}$ being released $q+1$ time units later.

Second, due to the offset, it may be impossible for a task $\tau_{p}$ to be executed inside a busy period of length $t$ even if $t \geq D_{p}$. To account for this behavior we need to define a new dynamic preemption level. In the following definition, $\mathcal{T}_{i}^{\prime}$ is the task set from Definition 1.

Definition 5. Given task set $\mathcal{T}_{i}^{\prime}$, we define the following dynamic preemption level:

$$
\pi_{i}(t)=\min _{j}\left(\left\{\pi_{j} \mid \phi_{j}^{\prime}+D_{j} \leq t\right\} \cup \pi_{s}\right)
$$

We can finally define the maximum dynamic blocking time for asynchronous task sets.

Definition 6. Given task set $\mathcal{T}_{i}^{\prime}$, the maximum dynamic blocking time is defined as:

$$
B_{i}(t)=\max _{j k}\left(\left\{C_{j k}-1 \mid D_{j}>\left(t+\Delta_{j i}^{\psi_{j k}}\right) \wedge \operatorname{ceil}\left(\rho_{j k}\right) \geq \pi_{i}(t)\right\} \cup 0\right)
$$

Lemma 10. $B_{i}\left(t_{2}-t_{1}\right)$ is an upper bound to the maximum blocking time experienced by tasks in a busy period $\left[t_{1}, t_{2}\right)$, where $t_{1}$ corresponds to an activation time of task $\tau_{i}$ and $t_{2}$ to the deadline of some task.

Proof: The proof is an extension of the proof of Lemma 8. As before, suppose that a job of task $\tau_{j}$ blocks higher priority tasks executed inside the busy period $(\operatorname{set} \mathcal{A})$.

Once again, $\tau_{j}$ can enter some critical section $\xi_{j k}$ at worst at $t_{1}-1$, so that the maximum blocking time can be no longer than $C_{j k}-1$. Furthermore, $\tau_{j}$ must be activated at least $\psi_{j k}+1$ time units before $t_{1}$ and its deadline must be greater than $t_{2}$, thus it must hold $D_{j}>t_{2}-t_{1}+\Delta_{j i}^{\psi_{j k}}$. Finally, since $\tau_{j}$ must be able to block some job in $\mathcal{A}$, it must also hold ceil $\left(\rho_{i j}\right) \geq \pi_{i}(t)$. In fact, no task $\tau_{p}$ in $\mathcal{A}$ can have a preemption level smaller than $\pi_{i}(t)$, since $\phi_{p}^{\prime}$ is the smallest time distance after $t_{1}$ at which $\tau_{p}$ can be activated. Therefore $B_{i}\left(t_{2}-t_{1}\right)$ is an upper bound to the maximum blocking time.

We can now introduce the main theorem. 
Theorem 6. Given task set $\mathcal{T}$ with $U \leq 1$, if $\forall 1 \leq i \leq N, \forall L \leq L^{\star}$ :

$$
d f(0, L)+B_{i}(L) \leq L
$$

where $L$ is an absolute deadline of task set $\mathcal{T}_{i}^{\prime}$ and $L^{\star}$ is the first idle time in the schedule of $\mathcal{T}_{i}^{\prime}$, then $\mathcal{T}$ is feasible.

Proof: The theorem is a straightforward extension of Theorem 2.

\subsection{Busy Period Length}

Note that the recurrence over the length of the busy period for task set $\mathcal{T}_{i}^{\prime}$ :

$$
L^{\star}=\sum_{j=1}^{N}\left(\left\lceil\frac{L^{\star}-\phi_{j}^{\prime}}{T_{j}}\right\rceil\right)_{0} C_{j}
$$

is no longer valid when blocking times are considered. In fact, a task $\tau_{j}$ whose offset is greater than or equal to the maximum length computed in this way, may still contribute to the busy period by adding a blocking time. The recurrence must thus be modified by adding the blocking time contribution:

$$
L^{\star}=\sum_{j=1}^{N}\left\lceil\frac{L^{\star}-\phi_{j}^{\prime}}{T_{j}}\right\rceil C_{j}+B_{j}^{\star}\left(L^{\star}\right)
$$

where

$$
B_{i}^{\star}(t)=\max \left(\left\{C_{j k}-1 \mid \phi_{j}^{\prime} \geq t \wedge \operatorname{ceil}\left(\rho_{j k}\right) \geq \pi_{i}^{\star}(t)\right\} \cup 0\right)
$$

and

$$
\pi_{i}^{\star}(t)=\min \left(\left\{\pi_{j} \mid \phi_{j}^{\prime}<t\right\} \cup \pi_{s}\right)
$$

In other words, we must consider the blocking time introduced by tasks with offset greater or equal to the currently computed length, due to critical sections that may introduce blocking on tasks with offsets smaller than the busy period length.

\section{Experimental Evaluation}

In this section, we evaluate the effectiveness of the proposed tests against Baruah's sufficient synchronous test described by Theorem 1 and against the exponential test executed by checking the demand function for every deadline until $\Phi+2 H$. For each experiment, we generated 2000 synthetic periodic task sets consisting of 6,10 and 20 tasks, respectively, and with total utilization ranging from 0.8 to 1 . No resource dependency is considered. 
Each task set was generated in the following way. First, utilizations $U_{i}$ were randomly generated according to a uniform distribution, so that the total utilization summed up to the desired value. Then periods were generated uniformly between 10 and 200 and the worst-case computation time of each task was computed based on utilization and period. Finally, relative deadlines were assigned to be either between 0.3 and 0.8 times the task's period or between half period and the period, and offsets were randomly generated between 0 and the period.

We experimented with two types of task sets. In the first case, we generated the periods so that the greatest common divisor between any two of them were a multiple of 5. In the second case, we chose the gcds as multiples of 10 . The basic idea is that, if the gcd between two periods is 1 , the distance between the release times of the two tasks can assume any value, 0 included. In the limit case in which all periods are relatively prime, it is possible to show that the synchronous test is necessary and sufficient also for asynchronous task sets. Note that in the real world, a situation in which the task periods are relatively prime is not very common.

In each experiment we computed the percentage of detected feasible task sets out of the total number of feasible task sets using Baruah's synchronous test and our test with one, two and three fixed tasks. In the following we will denote these tests with sync, 1-fixed, 2-fixed, 3-fixed, respectively. The results are presented in Figures 8-13.

Figures 8-10 shows the results for 6 tasks, with a minimum gcd of 10 in Figures 8 and 9 and a minimum gcd of 5 in Figure 10. In the first two figures, 1-fixed accepts a number of task sets up to $20 \%$ higher then the sync test. Performances are clearly lower with gcd $=5$, as shown in Figure 10. Also note that the 2-fixed test does not achieve significant improvements over the 1-fixed test, while the 3-fixed accepts up to $10 \%$ more task sets. Results are partially different in Figures 11 and 12 where we show the results for 10 tasks and gcd $=10$. The 1-fixed test again achieves good results, but the 2-fixed and particularly the 3 -fixed tests seem much less beneficial. In fact, increasing the number

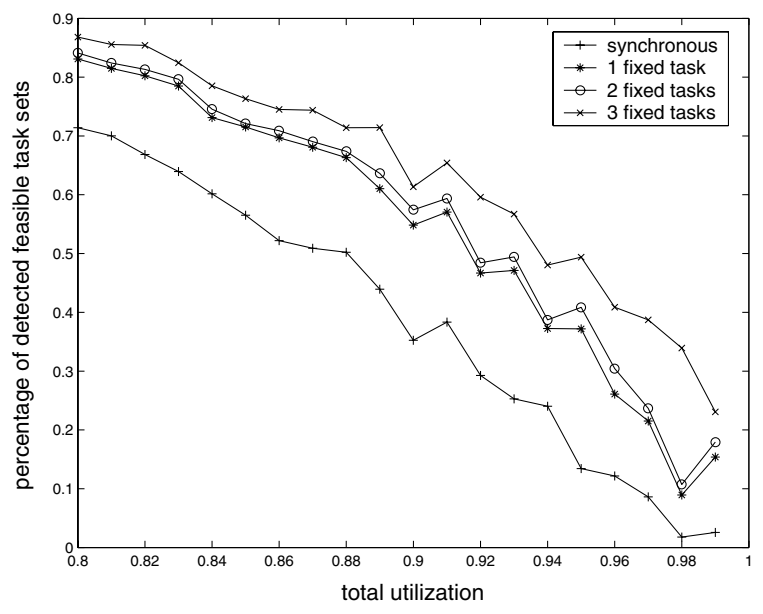

Figure 8. 6 tasks, gcd $=10$, deadline $\in[0.3,0.8] T$. 


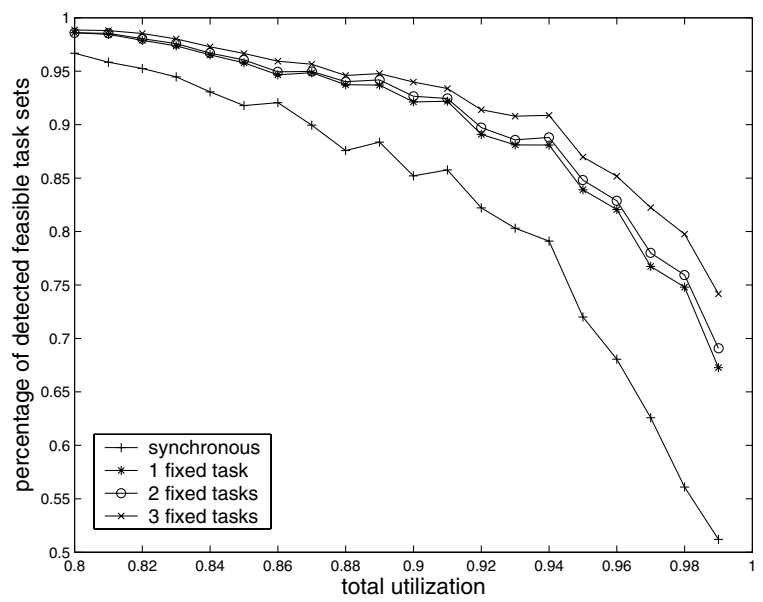

Figure 9. 6 tasks, gcd $=10$, deadline $\in[0.5,1.0] T$.

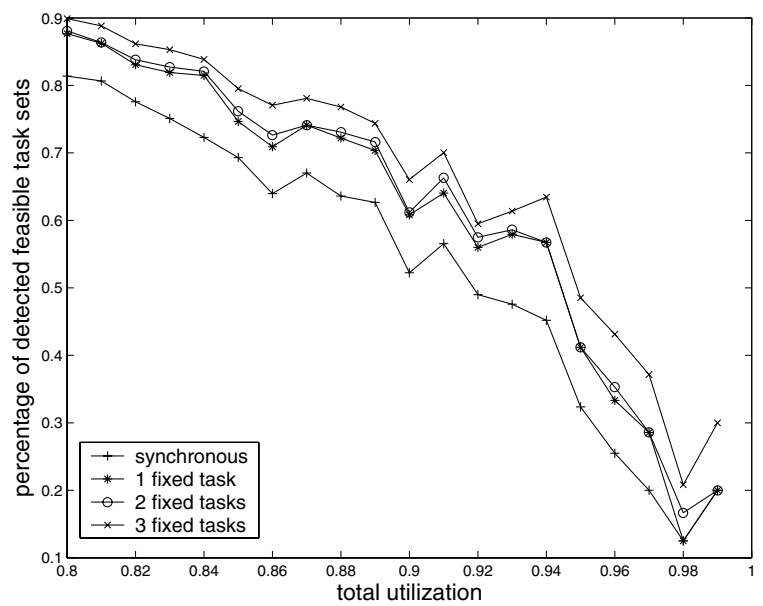

Figure 10. 6 tasks, $\operatorname{gcd}=5$, deadline $\in[0.3,0.8] T$.

of fixed tasks seems to be beneficial only if the number of fixed tasks $M$ is comparable to the number of total task $N$, but in that case the test obviously becomes not tractable (note that for $M=N-1$ the test is equivalent to the exponential one). The same holds in Figure 13 for 20 tasks and gcd $=10$; experiments with larger task sets lead to results that are quite similar to the ones in Figure 13, except for the performance of 2-fixed and 3 -fixed that becomes even more negligible with respect to 1-fixed, and are therefore not reported.

We also computed the mean number of simulation cycles needed to test a task set. Table 1 shows the results for gcd $=10$ and deadline between 0.3 and 0.8 times the period for the sync, 1-fixed and exponential test. Notice that the number of cycles needed for the 1-fixed 


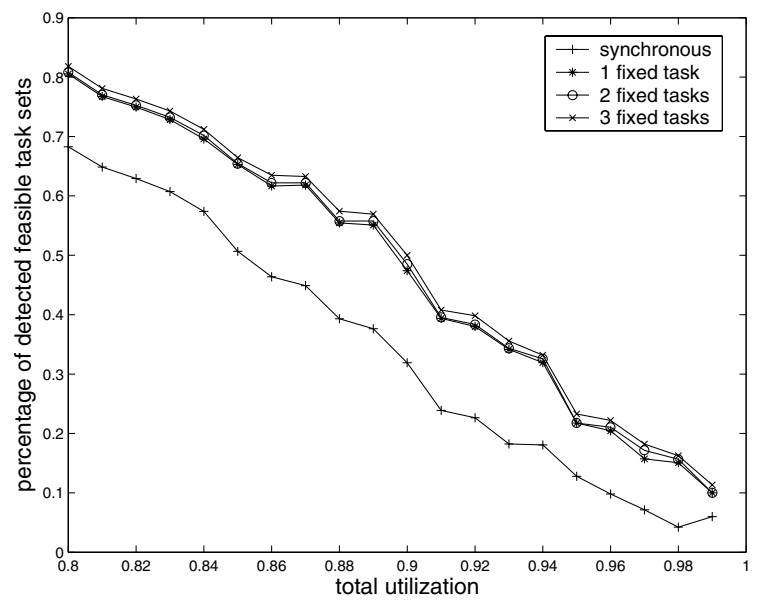

Figure 11. 10 tasks, $\mathrm{gcd}=10$, deadline $\in[0.3,0.8] T$.

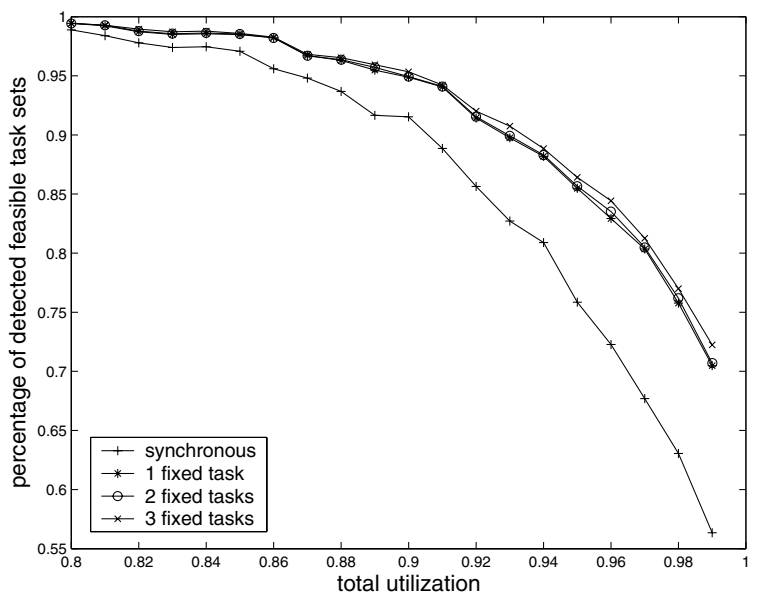

Figure 12. 10 tasks, gcd $=10$, deadline $\in[0.5,1.0] T$.

test is only one order of magnitude greater than the sync test. As the number of tasks increases we can appreciate that the growth in the number of cycles for the 1-fixed test is still acceptable compared to the corresponding growth of the exponential test.

\section{Related work}

The problem of feasibility analysis of asynchronous task sets can be found in many practical applications. For example, in distributed systems a transaction consists of a chain of precedence constrained tasks that must execute one after the other and each task can be allocated to a different processor. A transaction is usually modeled as a set of tasks with 
Table 1. Mean number of cycles, gcd $=10$, deadline $\epsilon$ $[0.3,0.8] T$.

\begin{tabular}{rccr}
\hline & Synchronous & 1 fixed task & Exponential \\
\hline 6 tasks & 40 & 67 & 2233 \\
10 tasks & 122 & 387 & 461356 \\
20 tasks & 639 & 6341 & 42781200 \\
\hline
\end{tabular}

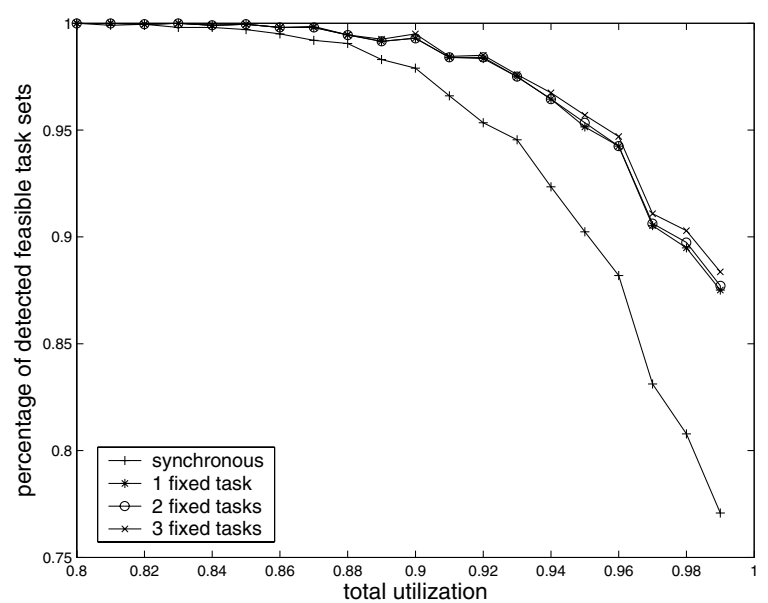

Figure 13. 20 tasks, $\mathrm{gcd}=10$, deadline $\in[0.5,1.0] T$.

offsets, such that the first task in the chain has offset 0 , the second task has an offset equal to the minimal response time of the first task, and so on. A task is further defined by a start time jitter. The start time jitter is the difference between the release time and the first activation time of a job. In this way, the problem of feasibility analysis of the entire system is divided into the problem of testing the feasibility of an asynchronous task set on each node.

In Spuri (1996a), the author developed a technique for computing the worst-case response time of tasks subject to start time jitter in synchronous task sets scheduled under EDF. An holistic analysis (Tindell et al., 1994; Spuri, 1996b) is then used to iteratively compute the worst case response time of each task and update the start time jitter of the next task in the chain, until the method converges to a result. The methodology has been recently extended in Palencia and González Harbour (2003) to transactions with offsets. However, while offsets are considered between tasks of the same transaction, no release time dependencies are considered between tasks of different transactions. In other words, the initial offset of each transaction can be arbitrarily moved.

The tests proposed in this paper are thus not directly comparable to the analysis developed in Palencia and González Harbour (2003) because of the different task model. However, it is possible to extend the proposed tests in order to account for the presence of transactions. This extension has been the topic of recent work (Pellizzoni and Lipari, 2005). 


\section{Conclusions and Future Work}

In this paper, we presented a new sufficient feasibility test for asynchronous task sets and proved it correct. Our test tries to take into account the offsets by computing the minimum distances between the release times of any two tasks. By analyzing a reduced set of critical arrival patterns, the proposed test keeps the complexity low and reduces the pessimism of the synchronous sufficient test. We showed, with an extensive set of experiments, that our test outperforms the synchronous sufficient test.

As future work, we are planning to extend our test to the case of relative deadline greater than the period. We would also like to explore the application of our test to the problem of minimizing the output jitter of a set of tasks. The output jitter is defined as the distance between the response time of two consecutive instances of a periodic task. Minimizing the output jitter is a very important issue in control systems (Cervin, 1999). In Baruah et al. (1999) a method to reduce the output jitter is presented, consisting in reducing as much as it is possible the relative deadlines of the tasks without violating the feasibility of the task set. However, in Baruah's work tasks are considered synchronous. In our opinion, the method can be improved by introducing appropriate offsets, with a beneficial effect on the output jitter.

\section{Acknowledgments}

This work has been partially supported by the Italian Ministry of University and Research within the COFIN 2001 project "Quack: a platform for the quality of new generation integrated embedded systems", and by the European Community within the IST project 2001-34820 ARTIST.

\section{References}

Baker, T. 1991. Stack-based scheduling of real-time processes. Journal of Real-Time Systems 3.

Baruah, S., Buttazzo, G., Gorinsky, S., and Lipari, G. 1999. Scheduling periodic task systems to minimize output jitter. In Proceedings of the International Conference on Real-Time Computing Systems and Applications. Hong Kong, pp. 62-69.

Baruah, S., Mok, A., and Rosier, L. 1990a. Preemptively scheduling hard-real-time sporadic tasks on one processor. In Proceedings of the 11th IEEE Real-Time Systems Symposium. pp. 182-190.

Baruah, S., Rosier, L., and Howell, R. 1990b. Algorithms and complexity concerning the preemptive scheduling of periodic real-time tasks on one processor. The Journal of Real-Time Systems 2.

Buttazzo, G. 1997. Hard Real-Time Computing Systems: Predictable Scheduling Algorithms and Applications. Boston: Kluwer Academic Publishers.

Cervin, A. 1999. Improved scheduling of control tasks. In Proceedings of the 11th Euromicro Conference on Real-Time Systems. York, UK, pp. 4-10.

Chen, M., and Lin, K. 1990. Dynamic priority ceilings: A concurrency control protocol for real-time systems. Journal of Real-Time Systems 2.

Dertouzos, M. L. 1974. Control robotics: The procedural control of physical processes. Information Processing. Jeffay, K. 1992. Scheduling sporadic tasks with shared resources in hard-real-time systems. In Proceedings of the 13th IEEE Real-Time Systems Symposium. Phoenix, pp. 89-99.

Leung, J.-T., and Merril, M. 1980. A note on preemptive scheduling of periodic real-time tasks. Information Processing Letters 3(11). 
Lipari, G. and Buttazzo, G. 2000. Schedulability analysis of periodic and aperiodic tasks with resource constraints. System Architecture 46: 327-338.

Liu, C. and Layland, J. 1973. Scheduling algorithms for multiprogramming in a hard-real-time environment. Journal of the Association for Computing Machinery 20(1).

Palencia, J., and González Harbour, M. 2003. Offset-based response time analysis of distributed systems scheduled under EDF. In 15th Euromicro Conference on Real-Time Systems. Porto, Portugal.

Pellizzoni, R. and Lipari, G. 2005. Improved schedulability analysis of real-time transactions with earliest deadline scheduling. In Proceedings of the 11th IEEE Real-Time and Embedded Technology and Applications Symposium. San Francisco, California.

Spuri, M. 1996a. Analysis of deadline scheduled real-time systems. Technical Report RR-2772, INRIA, France. Spuri, M. 1996b. Holistic analysis for deadline scheduled real-time distributed systems. Technical Report RR-2873, INRIA, France.

Tindell, K., Burns, A., and Wellings, A. 1994. An extendible approach for analysing fixed priority hard real-time tasks. Journal of Real Time Systems 6(2): 133-151.
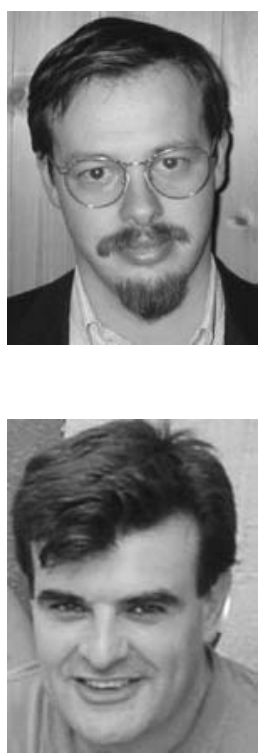

Rodolfo Pellizzoni received the Laurea degree in Computer Engineering from the "Università di Pisa" and the Diploma degree from the Scuola Superiore Sant'Anna, in 2004. He is presently a Ph.D. student in the Department of Computer Science at the University of Illinois at Urbana-Champaign. His main research interests are in real-time operating systems, scheduling theory and resource-allocation in distributed and multiprocessor systems.

Giuseppe Lipari graduated in Computer Engineering at the University of Pisa in 1996, and received the Ph.D. degree in Computer Engineering from Scuola Superiore Sant'Anna in 2000. During 1999, he was a visiting student at University of North Carolina at Chapel Hill, collaborating with professor S.K. Baruah and professor $\mathrm{K}$. Jeffay on real-time scheduling. Currently, he is assistant professor of Operating Systems with Scuola Superiore Sant'Anna. His main research activities are in real-time scheduling theory and its application to realtime operating systems, soft real-time systems for multimedia applications and component-based real-time systems. 